\title{
F

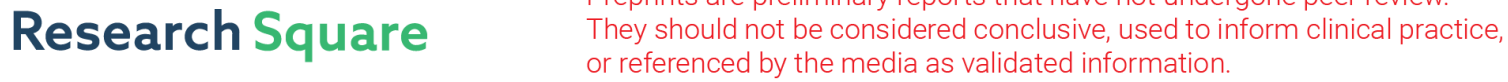 \\ GPX4 Plays a Crucial Role in Fuzheng Kang'ai Decoction-Induced Non-Small Cell Lung Cancer Cell Ferroptosis
}

\section{Yue-Yang Zhao}

Guangdong Provincial Hospital of Chinese Medicine, Guangzhou University of Chinese Medicine

\section{Yu-Qi Yang}

Guangdong Provincial Hospital of Chinese Medicine, Guangzhou University of Chinese Medicine Hong-Hao Sheng

Guangdong Provincial Hospital of Chinese Medicine, Guangzhou University of Chinese Medicine

\section{Qing Tang}

Guangdong Provincial Hospital of Chinese Medicine, Guangzhou University of Chinese Medicine

\section{Ling Han}

Guangdong Provincial Hospital of Chinese Medicine, Guangzhou University of Chinese Medicine

\section{Su-Mei Wang ( $\square$ wangsumei@gzucm.edu.cn )}

Guangdong Provincial Hospital of Chinese Medicine, Guangzhou University of Chinese Medicine

\section{Wan-Yin Wu}

Guangdong Provincial Hospital of Chinese Medicine, Guangzhou University of Chinese Medicine

\section{Research Article}

Keywords: FZKA, NSCLC, ferroptosis, GPX4

Posted Date: January 5th, 2022

DOl: https://doi.org/10.21203/rs.3.rs-1176282/v1

License: (c) (1) This work is licensed under a Creative Commons Attribution 4.0 International License. Read Full License 


\section{Abstract}

Background: Fuzheng Kang'ai decoction (FZKA) has been widely used to treat Non-Small Cell Lung Cancer (NSCLC) patients in China for decades, showing definite curative effects in clinic. Recently, we found that FZKA could induce NSCLC cell ferroptosis, another type of programmed cell death (PCD), which is totally different from cell apoptosis. Therefore, in the present study, we aim to discover the exact mechanism by which FZKA induces NSCLC cell ferroptosis, which is rarely studied in Traditional Chinese Medicine (TCM).

Methods: Cell counting kit-8 assay and EdU proliferation assay were performed to detect the cell viability. Cell ferroptosis triggered by FZKA was observed by performing lipid peroxidation assay, $\mathrm{Fe}^{2+}$ Ions assay, and mitochondrial ultrastructure by transmission electron microscopy. Ferroptosis inhibitors including liproxstatin-1 and UAMC 3203 were used to block ferroptosis. The ratio of GSH/GSSG was done to measure the alteration of oxidative stress. Western blot and qRT-PCR were carried out to detect the expression of SLC7A11, SLC3A2, and glutathione peroxidase 4 (GPX4) at protein and mRNA levels, respectively. Lentivirus transfection was performed to overexpress GPX4 stably. Animal model was done to verify the effect of FZKA-induced ferroptosis in NSCLC in vivo and immunohistochemistry was done to detect the expression of SLC7A11, SLC3A2 and GPX4 at protein level.

Results: First of all, in vitro experiments confirmed the inhibition effect of FZKA on NSCLC cell growth. We then, for the first time, found that FZKA induced NSCLC cell ferroptosis evidently, by increasing lipid peroxidation and cellular $\mathrm{Fe}^{2+}$ Ions. Moreover, characteristic morphological changes of NSCLC cell ferroptosis was observed under transmission electron microscopy. Mechanistically, GPX4, as a key inhibitor of lipid peroxidation, was greatly suppressed by FZKA treatment both at protein and mRNA levels. Furthermore, system xc' (SLC7A11 and SLC3A2) were found to be suppressed and a decreased GSH/GSSG ratio was observed at the same time by treating with FZKA. Notably, overexpressing GPX4 reversed the effect of FZKA-induced NSCLC cell ferroptosis significantly. Finally, the above effect was validated using animal model in vivo.

Conclusion: Our findings conclude that GPX4 plays a crucial role in FZKA-induced NSCLC cell ferroptosis, providing a novel molecular mechanism by which FZKA treats NSCLC.

\section{Background}

The burden of cancer incidence and mortality is rapidly growing worldwide. Lung cancer remained the leading cause of cancer death, with an estimated 1.8 million death in 2020 [1]. It is a serious threat to human health. NSCLC is the most common type of lung cancer, accounting for about $85 \%$ of all lung malignancies [2]. Approximately $70 \%$ of NSCLC is topically advanced or metastatic at the time of diagnosis [3]. Until the last decade, the 5-year overall survival rate for patients with metastatic NSCLC was less than 5\% [4]. Improved understanding of the biology of lung cancer had resulted in the development of new biomarker-targeted therapies and led to improvements for patients with advanced or metastatic 
cancers [5-7]. Disease progression is unavoidable in the advanced stage, thus additional strategies to extend survival and improve quality of life (QoL) are required. The Chinese herbal medicine (CHM) has been commonly used in cancer treatment as an adjuvant therapy in many countries, especially in China. It has been found that $\mathrm{CHM}$ has potential benefit in retarding tumor progression $[8,9]$.

FZKA, a formular containing $12 \mathrm{CHMs}$, has been confirmed to have definige benefit for treating NSCLC patients. In our previous study, we found that FZKA combined with geftinib could prolong progressionfree survival (PFS) and reduce the toxic effect, comparing with geftinib alone [10]. In addition, FZKA could also enhance the disease control rate (DCR) as well as median survival time (MST) of NSCLC patients $[11,12]$. Our further basic research showed that FZKA could inhibit NSCLC cell proliferation and promote cell apoptosis via AMPKa/IGFBP1/FOXO3a and STAT3/Bcl-2/Caspase-3 pathways, respectively $[13,14]$. Here in the present study, we, for the first time, found that FZKA could induce NSCLC cell ferroptosis.

Ferroptosis, first described in 2012 [15], is characterized by iron-dependent lipid peroxidation and metabolic constraints [16]. The happening of specific lipid peroxidation products directly precedes cellular disintegration and cell death [17]. Mechanistically, cysteine availability, glutathione (GSH) biosynthesis and proper functioning of GPX4 are crucial in the process of cell ferroptosis. GPX4 is a key inhibitor of lipid peroxidation. Ferroptotic cell death will be triggered on the condition of GPX4 inhibition. The importance of ferroptosis is the generation of specific phospholipid hydroperoxides in the presence of catalytically active iron, which is endogenously offset by the system xc/GSH/GPX4 axis [18-20]. System xc', composed of SLC7A11 and SLC3A2, is a cystine-glutamate anti-porter [21]. Therefore, disturbances in any of these protective compartments will result in ferroptotic cell death. In our study, we identified that system xc/GSH/GPX4 axis was involved in FZKA-induced NSCLC cell ferroptosis and GPX4 is the key molecular.

\section{Materials And Methods}

\section{Fuzheng Kang'ai decoction (FZKA)}

FZKA, containing 12 components, was obtained from Guangdong Kangmei Pharmaceutical Company Ltd (Guangdong, China), as previously reported [22]. The components of FZKA include Radix Pseudostellariae 30g, Rhizoma Atractylodis Macrocephalae 15g, Milkvetch Root 30g, Hedyotis Difusa 30g, Solanum Nigrum 30g, Chinese Sage Herb 30g, Indian Iphigenia Bulb 30g, Coix Seed 30g, Akebia Trifoliata Koidz 30g, Snake Bubble llicifolius 30g, Curcuma Zedoaria 15g, Licorice 10g. For in vitro experiments, the granules were dissolved in RPMI- 1640 medium to a final concentration of $20 \mathrm{mg} / \mathrm{mL}$ and centrifuged at $14,000 \mathrm{rpm}$ for $10 \mathrm{~min}$; the supernatant was then filtered using $0.22 \mu \mathrm{m}$ filter before use and the $\mathrm{pH}$ value of the cultured cells with the media was adjusted to 7.2-7.4 after FZKA addition. For in vivo experiments, animals were treated with FZKA by intragastric administration.

\section{High performance liquid chromatography (HPLC)}


The initial batch to batch consistency study was performed using HPLC, as previously reported [13]. Briefly, the sample solutions were put into the HPLC system (250×4.6 mm, $5 \mu \mathrm{m}, \mathrm{ACE}$, Scotland). The mobile phase consisted of deionized water with $0.1 \%$ formic acid $(A)$ and acetonitrile with $0.1 \%$ formic acid (B). The gradient elution program was as follows: $5 \%$ B at $0-5 \mathrm{~min}, 5-20 \% \mathrm{~B}$ at $5-10 \mathrm{~min}, 20-40 \%$ $B$ at $10-15 \mathrm{~min}, 40-95 \% \mathrm{~B}$ at $15-40 \mathrm{~min}$, and $95-100 \% \mathrm{~B}$ at $40-45 \mathrm{~min}$. The flow rate was $1.0 \mathrm{ml} / \mathrm{min}$, and the detection wavelength was set at $280 \mathrm{~nm}$. The injection volume was $10 \mu \mathrm{L}$ and the column temperature was maintained at $30^{\circ} \mathrm{C}$. The efficacy of different batch of FZKA is dependable [23] .

\section{Cell lines, reagents and antibodies}

NSCLC cell lines including A549, H1299, PC9 and H1650 were obtained from Guangzhou Cellcook Biotech Co. (Guangzhou, China). All cells were grown at $37^{\circ} \mathrm{C}$ in a humidified $5 \% \mathrm{CO}_{2}$ and $95 \%$ air and cultured in RPMI-1640 medium (Life Technologies, Carlsbad, CA, USA) containing 10\% FBS (Gibco, USA) and $0.5 \%$ penicillin-streptomycin sulfate (Invitrogen Life Technologies, Carlsbad, CA, USA). Annexin VFITC Apoptosis Detection Kit and Cell Counting Kit (CCK-8) were purchased from Shanghai Yisheng Biotechnology Co. (Shanghai, China). BODIPY ${ }^{\mathrm{TM}}$ 581/591 C11 was obtained from Thermo Fisher Scientific (Waltham, MA). Lentiviral vectors for overexpression constructs were purchased from GeneCopoeia (Rockville, USA). The antibodies were obtained from the following sources: GPX4 (ab125066) and GAPDH (ab9485) were purchased from Abcam (Cambridge, UK); SLC3A2 (4F2hc/CD98) (47213S), SLC7A11 (12691S), horseradish peroxidase (HRP) -conjugated goat anti-rabbit antibody (7074S), were from Cell Signalling Technology (Danvers, MA); SLC7A11 (bs-6883R) for immunohistochemistry was obtained from Bioss Biological Technology Co. Ltd. (Beijing, China). FerroOrange and GSSG/GSH Quantification Kit were purchased from Dojindo Molecular Technologies Company (Kumamoto, Japan).

\section{Cell counting kit-8 (CCK-8) assay}

Cell proliferation was measured by CCK-8 assay according to the manufacturer's protocol. Briefly, the NSCLC cells were administered with different treatments, and incubated with the CCK-8 reaction solution for $1.5 \mathrm{~h}$. After that, the optical density (OD) values were measured at the wavelength of $450 \mathrm{~nm}$ to evaluate cell viability.

\section{EdU proliferation assay}

5-ethynyl-2'-deoxyuridine (EdU) proliferation assay was performed to measure cell proliferation. Cells were plated in 96-well plates at a density of $8 \times 10^{3}$ cells/well. After adding FZKA for 24 hours, cells were treated with $50 \mu \mathrm{M}$ EdU (RiboBio, Guangzhou, China) and fixed with $4 \%$ paraformaldehyde in PBS for 30 min. After permeabilization with $0.5 \%$ TritonX-100 for $10 \mathrm{~min}$, the cells were stained with $1 \times$ Apollo reaction reagent. Then the DNA contents were stained with Hoechst 33342 for 30 min. The photographs were obtained using fluorescence microscope.

\section{Flow cytometry of cell death distribution}


Cells were treated with FZKA and ferroptosis inhibitors. Annexin V-FITC Apoptosis Detection Kit was used to observe the quadrant distribution of cell death. Both floating and adherent cells were collected and washed 3 times with PBS. Finally, $10 \mu \mathrm{L}$ Annexin V-FITC and $5 \mu \mathrm{L}$ PI were added into the cells at room temperature for $15 \mathrm{~min}$. The quadrant distribution of cell death was measured using flow cytometry with the acquisition criteria of 10000 events for each sample.

\section{Transmission electron microscopy}

The mitochondrial ultrastructure was observed by transmission electron microscopy (TEM). $2 \times 10^{6}$ cells were seeded into $100 \mathrm{~mm}$ cell culture dishes and exposed to FZKA decoction and erastin for $12 \mathrm{~h}$, respectively. After that, cells were collected, washed three times with PBS, and fixed with $2.5 \%$ glutaraldehyde. Samples were then pretreated according to standard procedures, including staining, dehydration, embedding, and slicing to obtain ultra-thin sections. During the analysis, images were acquired using a HITACHIH-7650 transmission electron microscope (Hitachi, Tokyo, Japan).

\section{Lipid peroxidation measurement}

C11-BODIPY $(10 \mu \mathrm{M})$ was added to FZKA treated or untreated cells for $0.5 \mathrm{~h}$, then cells were collected by trypsin. Oxidation of the poly-unsaturated butadienyl portion of C11-BODIPY resulted in a shift of the fluorescence emission peak from $\sim 590 \mathrm{~nm}$ to $\sim 510 \mathrm{~nm}$. Samples were analyzed using flow cytometry (Exc: $488 \mathrm{~nm}$, Em: $510 \mathrm{~nm}$ ) after washing twice with PBS, and the results were analyzed by NovoExpress software.

\section{Detection of cellular $\mathrm{Fe}^{2+}$ ions generation}

To clarify $\mathrm{Fe}^{2+}$ ions generation via the nanoparticles in cells, FerroOrange $\left(1 \mu \mathrm{M}\right.$, an intracellular Fe $\mathrm{Fe}^{2+}$ ions probe, Ex: $543 \mathrm{~nm}$, Em: $580 \mathrm{~nm}$ ) dispersed in serum-free medium was added to the cells, and cells were incubated for $30 \mathrm{~min}$ in a $37^{\circ} \mathrm{C}$ incubator. Cells were then collected by trypsin. Finally, the fluorescence of cells were captured using flow cytometry after washing twice with PBS.

\section{Lentivirus transfection}

Lentiviral vectors including GPX4 (CS-M0369-Lv105) and Negative Control (EX-NEG-Lv105) were purchased from GeneCopoeia. For lentivirus production, HEK293T packaging cells were transfected with $10 \mu \mathrm{g}$ lentiviral vectors using the calcium phosphate method. After 48 hours of incubation, the viral supernatant was collected and filtered. NSCLC cells were incubated overnight with the viral supernatant and supplemented with $10 \mu \mathrm{g} / \mathrm{ml}$ polybrene. Puromycin at a dose of $2 \mu \mathrm{g} / \mathrm{ml}$ was used to select the cell line overexpressing GPX4 stably.

\section{Western blot analysis}

Western blot was conducted as previously reported [14]. Briefly, the cells were harvested, washed and lysed with $1 \times$ RIPA buffer, and their protein concentrations were measured using Bradford method. SDS- 
PAGE was used to separate the protein in each sample. Proteins were transferred from gel to membrane. Then, the membrane was blocked and incubated with indicated primary antibodies. The blots were rinsed before probed with secondary antibodies. The reactive bands were visualized by ECL and scanned using the Bio-Rad ChemiDoc XRS+ Chemiluminescence imaging system (Bio-Rad, Hercules, CA, USA). All the results were analyzed by Image $\mathrm{J}$ software.

\section{Quantitative real-time PCR}

Total RNA was isolated using Trizol (Invitrogen, CA, USA). Transcriptor first strand cDNA synthesis kit (Roche, Basel, Switzerland) was used to convert RNAs to cDNAs. And FS essential DNA green master (Roche, Basel, Switzerland) was used to perform qRT-PCR. Complementary DNA from various cell samples was amplified with specific primers. GPX4: 5'-AGTGAGGCAAGACCGAAGT-3' and 5'AACTGGTTACACGGGAAGG-3'; GAPDH: 5'- GAACGGGAAGCTCACTGG -3' and 5'GCCTGCTTCACCACCTTCT -3 . Data were analyzed with $2^{-\triangle \triangle C t}$ for relative changes in gene expression.

\section{GSH/GSSG assay}

The intracellular level of GSH and GSSG were performed by GSSG/GSH Quantification Kit (Dojindo, Kumamoto, Japan), following the manufacturer's instruction. The concentration of total glutathione or GSSG was calculated via standard curve. GSH level was calculated as: GSH = (total glutathione-GSSG) $\times$ 2. The ratio of GSH/GSSG was calculated as [GSH]/ [GSSG].

\section{Animal model}

All animal experiments were approved by the Ethics Committee of Guangdong Provincial Hospital of Chinese Medicine (2020079). A total of $1.0 \times 10^{6}$ A549 cells were subcutaneously injected into the right flank of the athymic BALB/c nude mice (aged 4-6 weeks, weight 18-20 g, female; Vital River, Beijing, China). When the tumor mass became palpable (at day 4 after injection), the mice were randomly divided into three groups: control, FZKA $(31 \mathrm{~g} / \mathrm{kg})$ and combination with FZKA and liproxstatin$1(30 \mathrm{mg} / \mathrm{kg})$. Tumors were measured every 5 days with digital calipers. The tumor volume (in $\left.\mathrm{mm}^{3}\right)$ was calculated using the formula: Volume $=\left(L \times W^{2}\right) / 2$. Mice were sacrificed around day 25 after injection, when some of the tumors reached the size limit set by the institutional animal care and use committee. Tumors were weighed after careful resection.

\section{Immunohistochemistry}

The protein levels of GPX4, SLC7A11 and SLC3A2 expression were detected immunohistochemically on paraffin-embedded xenograft tumor tissue sections. Briefly, sections were treated with $10 \mathrm{mM}$ sodium citrate buffer $(\mathrm{pH} \mathrm{6.0)}$ for heat-induced retrieval of the antigen and immersed in $3 \%$ hydrogen peroxide solution to inhibit endogenous peroxidase activity, followed by incubation of the sections in $5 \%$ bovine serum albumin to block nonspecific binding. The sections were incubated with primary antibodies against GPX4 (1:250), SLC7A11 (1:100) and SLC3A2 (1:100) at $4^{\circ} \mathrm{C}$ overnight and then incubated with 
biotinylated secondary antibody followed by the Liquid DAB Substrate Chromogen System according to the manufacturer's instructions. Protein expression level was evaluated by counting at least 500 tumor cells in at least five representative high-power fields. The percentage of positive tumor cells and the staining intensity were multiplied to produce a weighted score for each case [24].

\section{Statistical analysis}

Statistical analysis was performed using the SPSS statistical software. Statistical evaluation for data analysis used Student's t-test when there were only two groups (two sided) and differences between groups were assessed by one-way ANOVA. All data are reported as Mean $\pm S D$. Differences between groups were considered significant statistically when $p \leq 0.05$.

\section{Results}

\section{NSCLC cell growth was inhibited by FZKA in vitro}

Our previous studies have shown that FZKA inhibited the growth of NSCLC cell lines including A549, PC9, and $\mathrm{H} 1975$ cells $[23,25]$. In the present study, we further observed the effect of FZKA on NSCLC cell growth inhibition in another NSCLC cell types including $\mathrm{H} 1650$ and $\mathrm{H} 1299$ using CCK-8 assay. We reconfirmed that FZKA decreased $\mathrm{H} 1650$ and $\mathrm{H} 1299$ cell viability in a dose- and time-dependent manner (Fig. 1A). Similar findings were also demonstrated by EdU incorporation assay, which detects 5-bromo-2'deoxyuridine (BrdU) incorporated into cellular DNA during cell proliferation using an anti-BrdU antibody (Fig. 1B). Intriguingly, we found that blocking ferroptosis by UAMC 3203 and liproxstatin-1 could significantly reversed changes in the quadrants of Annexin V-/PI+ (Q1) and Annexin V+/PI+ (Fig. 1C). The cells in Q1 quadrant in the top left had been supposed as non-apoptotic cells, and ferroptotic cell death was included in Q1 quadrant[26]. The data showed that inhibiting ferroptosis could decrease the percent of Q1 induced by FZKA, which suggests that ferroptosis plays an important role in the inhibition effect of FZKA in NSCLC cells and FZKA may promote ferroptosis in NSCLC cells.

\section{NSCLC cell ferroptosis was induced by FZKA}

To identify whether NSCLC cell ferroptosis was induced by FZKA treatment, lipid peroxidation and intracellular-free iron, as two key characteristics of cell ferroptosis, were then detected in NSCLC cells after treatment with FZKA [27]. C11-BODIPY was used as a lipid peroxidation probe in mammalian cells [28]. The intracellular labile Fe(II) levels in the living cells were measured by FerroOrange [29]. The results showed that FZKA increased the levels of lipid peroxidation (Fig.2A) and intracellular-free iron (Fig.2B) in A549 and PC9 cells. The same results were also observed in H1299 and H1650 cells (Fig.S1). Moreover, the characteristic changes of ferroptosis on mitochondria, including decreased cristae, shrunken mitochondria, and increased membrane density $\llbracket$ were further observed under TEM in NSCLC cells (Fig.2C, Fig. S2).

\section{Blocking ferroptosis reversed the inhibition effect of FZKA on NSCLC cells}


To further observe the role of ferroptosis in FZKA-treated NSCLC cells, two ferroptosis inhibitors including UAMC 3203 and liproxstatin-1 were applied to block ferroptosis. As shown in Figure 3A and S3, the FZKAinduced elevation of lipid peroxidation was almost reversed by treating with UAMC 3203 and liproxstatin1. Our further data showed that blocking ferroptosis remarkedly reversed the inhibition effect of FZKA on NSCLC cell lines (A549, PC9, H1650 and H1299), as shown in the Figure3B. These results indicated that ferroptosis plays a vital role in FZKA treated NSCLC cells.

\section{GPX4 was significantly suppressed by FZKA in NSCLC cells}

GPX4 is a key inhibitor of lipid peroxidation and ferroptosis. The down-regulation of GPX4 could directly or indirectly trigger ferroptosis as a result of lipid peroxidation inhibition. We detected the expressions of GPX4 at protein and mRNA level after treatment with FZKA. Our data found that the protein level of GPX4 was significantly decreased following the application of FZKA in NSCLC cells (Fig.4A). Meanwhile, the mRNA level of GPX4 were also decreased by treating with FZKA (Fig.4B). The above data indicated that GPX4 might be a main molecular in the FZKA-induced NSCLC cell ferroptosis process.

\section{System xc/GSH axis was involved in FZKA-induced NSCLC cell ferroptosis}

The cystine-glutamate antiporter system xc', which is composed of the subunits SLC7A11 and SLC3A2, plays a protective role against cell ferroptosis. We revealed that the protein levels of SLC7A11 and SLC3A2 were obviously decreased following the application of FZKA in a dose- dependent manner in $\mathrm{H} 1650$ and $\mathrm{H} 1299$ cells (Fig. 5A). Glutathione is a tripeptide that is derived from cysteine, glutamate, and glycine, among which cysteine is the rate-limiting precursor. As expected, the amount of GSH was significantly decreased by FZKA. GSH is highly reactive with lipid ROS, and their reaction generates glutathione disulfide (GSSG). A reduced ratio of GSH/GSSG is considered to be a marker of oxidative stress. In our study, we found that FZKA reduced the ratio of GSH/GSSG significantly (Fig.5B). System xc and GSH are at the upstream of GPX4, therefore, our data showed that system Xc/GSH/GPX4 axis plays an important role in FZKA-induced NSCLC cell ferroptsis.

\section{Over-expressing GPX4 reversed the effect of FZKA-induced cell ferroptosis}

Since GPX4 plays a crucial role in the process of cell ferroptosis, we were wondering whether GPX4 mediated the effect of FZKA-induced NSCLC ferroptosis. We then over-expressed GPX4 in H1299 and PC9 cells by transfecting lentivirus (Fig.6A). As expected, the induced effect of NSCLC ferroptosis by FZKA was substantially decreased following GPX4 overexpression as shown by lipid peroxidation assay (Fig.6B and Fig.S4). Interestingly, NSCLC cell viability inhibition by FZKA was also partially reversed when over-expressed GPX4 (Fig.6C). This data further confirmed that GPX4 contributes to the effect of FZKAinduced NSCLC cell ferroptosis and FZKA-suppressed NSCLC cell growth, suggesting that GPX4 plays a crucial role in FZKA-treated NSCLC cells.

\section{Blocking ferroptosis rescued the effect of FZKA-induced tumor growth inhibition in vivo}


To validate the effect of FZKA-induced NSCLC cell ferroptosis in vivo, we constructed NSCLC cell xenograft model. As shown in Figure 7A and 7B, mice tumor growth was obviously inhibited by FZKA treatment undoubtedly. Notably, when cell ferroptosis was blocked by liproxstatin-1, the inhibition effect of tumor growth by FZKA was significantly rescued. Then system $\mathrm{xc}^{-}$and GPX4 were detected by Western blot and immunohistochemistry. As expected, the data was consistent with in vitro results showing downregulated expression of system xc and GPX4 in the FZKA-treated group (Fig.7C and 7D). Totally, our xenagraft model data reconfirmed the effect of FZKA-induced NSCLC cell ferroptosis and system Xc/GPX4 axis palys a crucial in the process.

\section{Discussion}

Ferroptosis, as another type of PCD, is entirely different from cell apoptosis, necroptosis, autophagic cell death and other forms of regulated necrotic cell death. The ferroptosis-induced cell death is characterized by iron-dependent lipid peroxidation $[15,16]$. Ferroptotic cell death was considered to locate at Q1 quadrant by flow cytometry of Annexin V/PI staining [26]. In our study, we found that blocking ferroptosis via ferroptosis inhibitors could reversed Q1 quadrant after FZKA treatment, indicating the important role of ferroptosis in FZKA-treated NSCLC cells. Since lipid peroxidation and intracellular-free iron are two key characteristics of cell ferroptosis [27], we then detected the lipid peroxidation and intracellular-free iron in NSCLC cells after treatment with FZKA. Our results showed that FZKA could increase lipid peroxidation and intracellular-free iron, indicating that FZKA might have the ability to induce NSCLC cell ferroptosis. We then observed characteristic changes on mitochondria of ferroptosis using TEM in NSCLC cells when treated with FZKA. Treatment with FZKA resulted in swollen mitochondria with fractured cristae and increased membrane density, which is consistent with erastin, a ferroptosis inducer. Therefore, our data provided solid evidences that FZKA induces NSCLC cell ferroptosis.

Early research indicated the primary role of GPX4 in protecting against oxidative damage [30,31]. Cells with GPX4 overexpression are resistant to lipid hydroperoxide-triggered cell death [32]. Later, more studies provided evidences that silencing GPX4 could invariably cause ferroptosis [33]. Some researches reported that GPX4 was decreased at protein levels, resulting in cell ferroptosis [34, 35]. In our study, the expression of GPX4 at the protein and mRNA levels were significantly suppressed by FZKA in NSCLC cells. Disturbances in any of these protective compartments inlucing system $\mathrm{Xc}^{-}$and $\mathrm{GSH}$ biosynthesis, upstream of GPX4, might result in ferroptotic cell death [36]. Our results showed that FZKA decreased the expression of SLC7A11 and SLC3A2 in NSCLC cells. And the ratio of GSH/GSSG was also suppressed in FZKA-treated group. Most importantly, when we over-expressed GPX4, it could reverse NSCLC cell ferroptosis induced by FZKA. And cell viability inhibition effect by FZKA was also partially reversed by over-expressing GPX4 at the same time. Therefore, our data indicated the critical role of GPX4 in the induction of NSCLC cell ferroptosis by FZKA. Our findings provide a valid evidence that FZKA might function as a GPX4 inhibitor in treating NSCLC patients, and system Xc/GSH/GPX4 axis was involved in the process. 


\section{Conclusions}

In our study, we investigated the effect of FZKA on NSCLC cell ferroptosis both in intro and in vivo. Our results showed that FZKA induces ferroptosis by suppressing GPX4 in NSCLC, which is rarely studied in the field of Traditional Chinese Medicine. We provides solid evidences to clarify why FZKA benefits NSCLC patients in clinic.

\section{Abbreviations}

FZKA: Fuzheng Kang'ai decoction; NSCLC: Non-Small Cell Lung Cancer; PCD: programmed cell death; TCM: Traditional Chinese Medicine; GPX4: glutathione peroxidase 4; QoL: quality of life; CHM: Chinese herbal medicine; PFS: progression-free survival; DCR: disease control rate; MST: median survival time; GSH: glutathione; TEM: transmission electron microscopy.

\section{Declarations}

\section{Ethics approval and consent to participate}

All animal experiments are followed the Guide for Care and Use of Laboratory Animals by America (National Institute of Health) and approved by the Ethics Committee of Guangdong Provincial Hospital of Chinese Medicine (2020079).

\section{Consent for publication}

All the authors are consent for the publication of this article.

\section{Availability of data and materials}

The datasets used and/or analyzed during the current study are available from the corresponding author on reasonable request.

\section{Competing interests}

The authors declare that they have no competing interests.

\section{Funding}

This work was supported by grants from the National Natural Science Foundation of China (81974543, 81903991), the Guangdong Natural Science Foundation of China (2019A1515011362, 2021A1515410007), the Guangzhou science and technology plan project (202002030155, 202102010160), the Scientific Research Project in Universities of Guangdong Provincial Department of Education (2020KTSCX029), the State Key laboratory of Dampness Syndrome of Chinese Medicine (SZ2021ZZ38), the Guangdong Provincial Key Laboratory of Clinical Research on Traditional Chinese Medicine Syndrome (ZH2020KF03), the Chinese medicine science and technology research project of 
Guangdong Provincial Hospital of Chinese Medicine (YN2019MJ09), the China Postdoctoral Science Foundation Project (2021M690796), and the Science and Technology Planning Project of Guangdong Province (2017B030314166).

\section{Authors' contributions}

Wanyin Wu (wwanyin@gzucm.edu.cn) was responsible for the project design. Sumei Wang (wangsumei@gzucm.edu.cn) was responsible for the experiment design and manuscript editing. Ling Han (linghan99@gzucm.edu.cn) provided some key suggestions. Yueyang Zhao (zyy6181@126.com) performed most of the experiments and wrote the manuscript draft. Yuqi

Yang (yyqlzy1122@gzucm.edu.cn) and Honghao Sheng (shh613828@163.com) performed some of the experiments. Qing Tang (tangqingyanjiu@163.com) provided some help for the animal experiments. All authors read and approved the final manuscript.

\section{Acknowledgments}

We thank Hongshen Wang for his helpful assistance on the immunohistochemistry of this study.

\section{Author details}

${ }^{1}$ Department of Hematology, Guangdong Provincial Hospital of Chinese Medicine, The Second Clinical Medical College, Guangzhou University of Chinese Medicine, Guangzhou, Guangdong 510120, China

2 Department of Oncology, Clinical and Basic Research Team of TCM Prevention and Treatment of NSCLC, The Second Clinical College of Guangzhou University of Chinese Medicine, Guangdong Provincial Hospital of Chinese Medicine, Guangzhou, Guangdong 510120, China ${ }^{3}$ The Postdoctoral Research Station, Guangzhou University of Chinese Medicine, Guangzhou, Guangdong 510120, China ${ }^{4}$ The Second Clinical Medical College, Guangzhou University of Chinese Medicine, The Second Affiliated Hospital of Guangzhou University of Chinese Medicine; Guangzhou, Guangdong 510120, China ${ }^{5}$ State Key laboratory of Dampness Syndrome of Chinese Medicine, The Second Affiliated Hospital of Guangzhou University of Chinese Medicine, Guangzhou, Guangdong 510120, China ${ }^{6}$ Guangdong Provincial Key Laboratory of Clinical Research on Traditional Chinese Medicine Syndrome; GuangdongHong Kong-Macau Joint Lab on Chinese Medicine and Immune Disease Research, Guangzhou University of Chinese Medicine, Guangzhou, Guangdong 510120, China.

\section{References}

1. Sung H, Ferlay J, Siegel RL, Laversanne M, Soerjomataram I, Jemal A, Bray F: Global Cancer Statistics 2020: GLOBOCAN Estimates of Incidence and Mortality Worldwide for 36 Cancers in 185 Countries. CA: a cancer journal for clinicians 2021, 71:209-249.

2. Herbst RS, Heymach JV, Lippman SM: Lung cancer. N Engl J Med 2008, 359:1367-1380. 
3. Molina JR, Yang P, Cassivi SD, Schild SE, Adjei AA: Non-small cell lung cancer: epidemiology, risk factors, treatment, and survivorship. Mayo Clin Proc 2008, 83:584-594.

4. Arbour KC, Riely GJ: Systemic Therapy for Locally Advanced and Metastatic Non-Small Cell Lung Cancer. JAMA 2019, 322:764.

5. Melosky B, Chu Q, Juergens RA, Leighl N, lonescu D, Tsao MS, McLeod D, Hirsh V: Breaking the biomarker code: PD-L1 expression and checkpoint inhibition in advanced NSCLC. Cancer Treat Rev 2018, 65:65-77.

6. Jordan EJ, Kim HR, Arcila ME, Barron D, Chakravarty D, Gao J, Chang MT, Ni A, Kundra R, Jonsson P, et al: Prospective Comprehensive Molecular Characterization of Lung Adenocarcinomas for Efficient Patient Ma tching to Approved and Emerging Therapies. Cancer Discov 2017, 7:596-609.

7. Zhu C, Zhuang W, Chen L, Yang W, Ou WB: Frontiers of ctDNA, targeted therapies, and immunotherapy in non-small-cell lung cancer. Trans/ Lung Cancer Res 2020, 9:111-138.

8. Wang S, Long S, Wu W: Application of Traditional Chinese Medicines as Personalized Therapy in Human Cancers. Am J Chin Med 2018, 46:953-970.

9. Luo H, Vong CT, Chen H, Gao Y, Lyu P, Qiu L, Zhao M, Liu Q, Cheng Z, Zou J, et al: Naturally occurring anti-cancer compounds: shining from Chinese herbal medicine. Chin Med 2019, 14:48.

10. Yang XB, Chai XS, Wu WY, Long SQ, Deng H, Pan ZQ, He WF, Zhou YS, Liao GY, Xiao SJ: Gefitinib plus Fuzheng Kang'ai Formula () in Patients with Advanced Non-Small Cell Lung Cancer with E pidermal Growth Factor Receptor Mutation: A Randomized Controlled Trial. Chin J Integr Med 2018, 24:734740 .

11. Wu WY, Yang XB, Deng H, Long SQ, Sun LS, He WF, Zhou YS, Liao GY, Chan SM, Shan SP: Treatment of advanced non-small cell lung cancer with extracorporeal high frequency thermotherapy com bined with Chinese medicine. Chin J Integr Med 2010, 16:406-410.

12. Yang XB, Wu WY, Long SQ, Deng H, Pan ZQ: Effect of gefitinib plus Chinese herbal medicine (CHM) in patients with advanced non-small-cell lung cancer: a retrospective case-control study. Complement Ther Med 2014, 22:1010-1018.

13. Zheng F, Wu J, Li X, Tang Q, Yang L, Yang X, Wu W, Hann SS: Chinese Herbal Medicine Fuzheng Kang-Ai Decoction Inhibited Lung Cancer Cell Growth through AMPKa-Med iated Induction and Interplay of IGFBP1 and FOXO3a. Evid Based Complement Alternat Med 2016, 2016:5060757.

14. Wang S, Long S, Xiao S, Wu W, Hann SS: Decoction of Chinese Herbal Medicine Fuzheng Kang-Ai Induces Lung Cancer Cell Apoptosis via STAT3/Bcl -2/Caspase-3 Pathway. Evid Based Complement Alternat Med 2018, 2018:8567905.

15. Dixon SJ, Lemberg KM, Lamprecht MR, Skouta R, Zaitsev EM, Gleason CE, Patel DN, Bauer AJ, Cantley AM, Yang WS, et al: Ferroptosis: an iron-dependent form of nonapoptotic cell death. Cell 2012, 149:1060-1072.

16. Seibt TM, Proneth B, Conrad M: Role of GPX4 in ferroptosis and its pharmacological implication. Free Radic Biol Med 2019, 133:144-152. 
17. Angeli J, Shah R, Pratt DA, Conrad M: Ferroptosis Inhibition: Mechanisms and Opportunities. Trends Pharmacol Sci 2017, 38:489-498.

18. Dixon SJ, Lemberg KM, Lamprecht MR, Skouta R, Zaitsev EM, Gleason CE, Patel DN, Bauer AJ, Cantley AM, Yang WS, et al: Ferroptosis: an iron-dependent form of nonapoptotic cell death. Cell 2012, 149:1060-1072.

19. Yang WS, SriRamaratnam R, Welsch ME, Shimada K, Skouta R, Viswanathan VS, Cheah JH, Clemons PA, Shamji AF, Clish CB, et al: Regulation of ferroptotic cancer cell death by GPX4. Cell 2014, 156:317-331.

20. Friedmann Angeli JP, Schneider M, Proneth B, Tyurina YY, Tyurin VA, Hammond VJ, Herbach N, Aichler M, Walch A, Eggenhofer $\mathrm{E}$, et al: Inactivation of the ferroptosis regulator Gpx4 triggers acute renal failure in mice. Nat Cell Biol 2014, 16:1180-1191.

21. Conrad M, Sato $H$ : The oxidative stress-inducible cystine/glutamate antiporter, system x (c) (-) : cystine supplier and beyond. Amino Acids 2012, 42:231-246.

22. Yang XB, Wu WY, Long SQ, Deng H, Pan ZQ: Effect of gefitinib plus Chinese herbal medicine (CHM) in patients with advanced non-small-cell lung cancer: a retrospective case-control study. Complement Ther Med 2014, 22:1010-1018.

23. Wang S, Peng Z, Li W, Long S, Xiao S, Wu W: Fuzheng Kang-Ai decoction enhances the effect of Gefitinib-induced cell apoptosis in lung cancer thro ugh mitochondrial pathway. Cancer Cell Int 2020, 20:185.

24. Lu CD, Altieri DC, Tanigawa N: Expression of a novel antiapoptosis gene, survivin, correlated with tumor cell apoptosis and p53 accu mulation in gastric carcinomas. Cancer Res 1998, 58:1808-1812.

25. Zheng F, Zhao Y, Li X, Tang Q, Wu J, Wu W, Hann SS: The repression and reciprocal interaction of DNA methyltransferase 1 and specificity protein 1 contri butes to the inhibition of MET expression by the combination of Chinese herbal medicine FZKA decocti on and erlotinib. J Ethnopharmacol 2019, 239:111928.

26. Gai C, Liu C, Wu X, Yu M, Zheng J, Zhang W, Lv S, Li W: MT1DP loaded by folate-modified liposomes sensitizes erastin-induced ferroptosis via regulating miR-3 65a-3p/NRF2 axis in non-small cell lung cancer cells. Cell Death Dis 2020, 11:751.

27. Lei P, Bai T, Sun Y: Mechanisms of Ferroptosis and Relations With Regulated Cell Death: A Review. Front Physiol 2019, 10:139.

28. Ortega Ferrusola C, González Fernández L, Morrell JM, Salazar Sandoval C, Macías García B, Rodríguez-Martinez H, Tapia JA, Pe A FJ: Lipid peroxidation, assessed with BODIPY-C11, increases after cryopreservation of stallion spermatozo $\mathrm{a}$, is stallion-dependent and is related to apoptotic-like changes. Reproduction 2009, 138:55-63.

29. Hirayama T, Niwa M, Hirosawa S, Nagasawa H: High-Throughput Screening for the Discovery of Iron Homeostasis Modulators Using an Extremely Sensiti ve Fluorescent Probe. ACS Sens 2020, 5:29502958. 
30. Imai H, Sumi D, Sakamoto H, Hanamoto A, Arai M, Chiba N, Nakagawa Y: Overexpression of phospholipid hydroperoxide glutathione peroxidase suppressed cell death due to oxid ative damage in rat basophile leukemia cells (RBL-2H3). Biochem Biophys Res Commun 1996, 222:432-438.

31. Yagi K, Komura S, Kojima H, Sun Q, Nagata N, Ohishi N, Nishikimi M: Expression of human phospholipid hydroperoxide glutathione peroxidase gene for protection of host cel Is from lipid hydroperoxide-mediated injury. Biochem Biophys Res Commun 1996, 219:486-491.

32. Geiger PG, Thomas JP, Girotti AW: Lethal damage to murine L1210 cells by exogenous lipid hydroperoxides: protective role of glutathione-dependent selenoperoxidases. Arch Biochem Biophys 1991, 288:671-680.

33. Maiorino M, Conrad M, Ursini F: GPx4, Lipid Peroxidation, and Cell Death: Discoveries, Rediscoveries, and Open Issues. Antioxid Redox Signal 2018, 29:61-74.

34. Zhang Y, Swanda RV, Nie L, Liu X, Wang C, Lee H, Lei G, Mao C, Koppula P, Cheng W, et al: mTORC1 couples cyst(e)ine availability with GPX4 protein synthesis and ferroptosis regulation. Nat Commun $2021,12: 1589$.

35. Lou JS, Zhao LP, Huang ZH, Chen XY, Xu JT, Tai WC, Tsim K, Chen YT, Xie T: Ginkgetin derived from Ginkgo biloba leaves enhances the therapeutic effect of cisplatin via ferropto sis-mediated disruption of the Nrf2/HO-1 axis in EGFR wild-type non-small-cell lung cancer. Phytomedicine 2021, 80:153370.

36. Lee N, Carlisle AE, Peppers A, Park SJ, Doshi MB, Spears ME, Kim D: xCT-Driven Expression of GPX4 Determines Sensitivity of Breast Cancer Cells to Ferroptosis Inducers. Antioxidants (Basel) 2021, 10.

\section{Figures}

\section{Figure 1}

\section{FZKA inhibited the growth of NSCLC cells in vitro.}

A, $\mathrm{H} 1650$ and $\mathrm{H} 1299$ cells were treated with different concentrations of FZKA for up to $72 \mathrm{~h}$. The cells were collected and processed for CCK-8 assay as described in the Materials and Methods section, ${ }^{*} p<0.05$. B, H1650 and $\mathrm{H} 1299$ cells were treated with FZKA ( $\left.1 \mathrm{mg} / \mathrm{ml}\right)$ for $24 \mathrm{~h}$, followed by colorimetric BrdU ELISA methods. C. Cultured A549 cells were treated with FZKA $(1.5 \mathrm{mg} / \mathrm{ml})$, in the presence and absence of ferroptosis inhibitors. Cell were stained with Annexin $\mathrm{V}$ and $\mathrm{PI}$ and analyzed by flow cytometry, ${ }^{*} p<0.05$. 
A
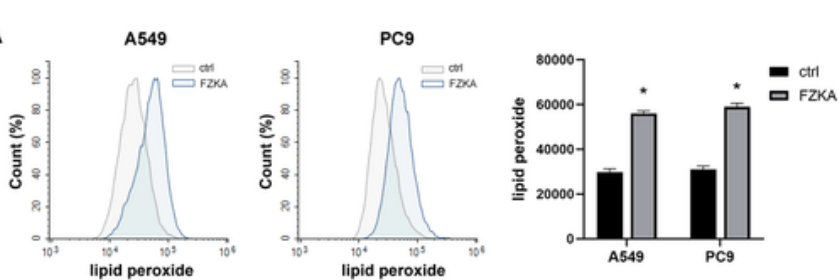

B
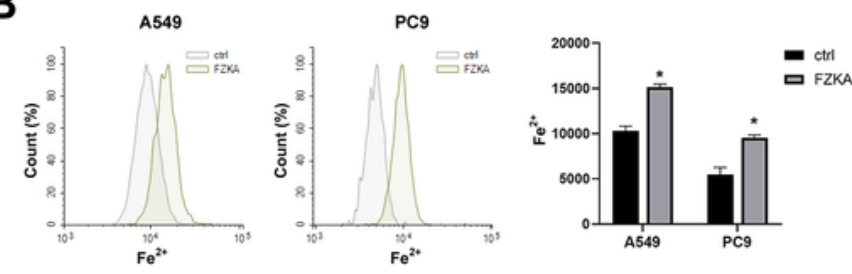

C

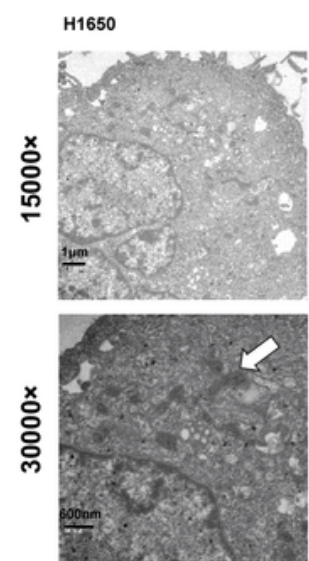

DMSO

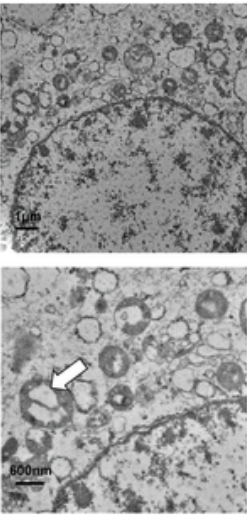

erastin
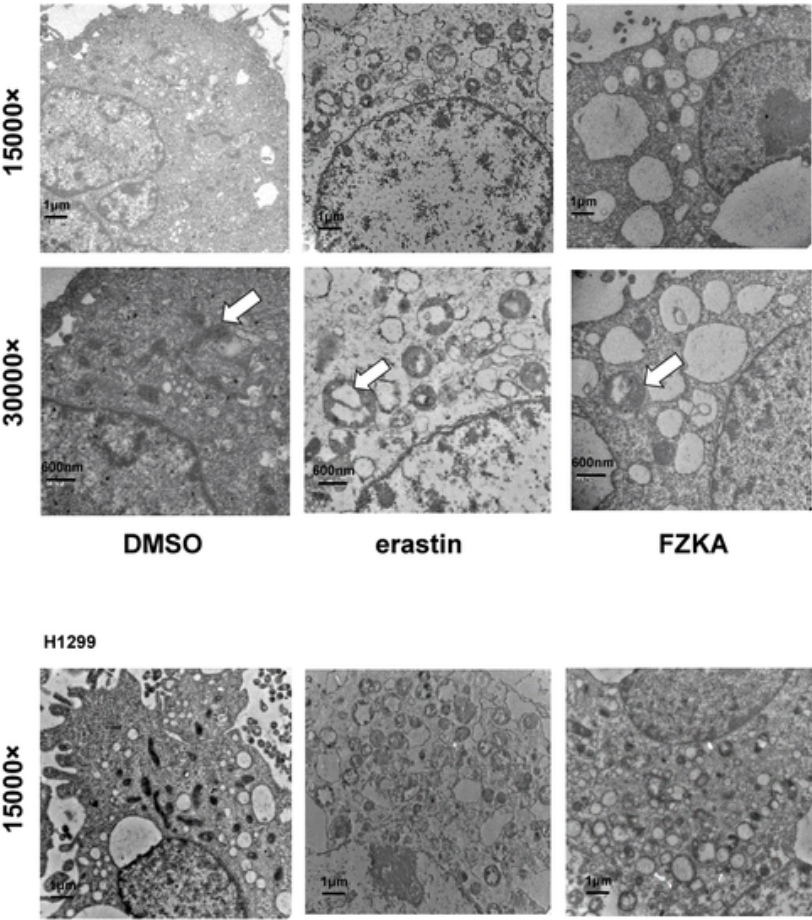

FZKA

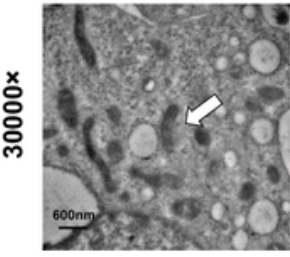

DMSO

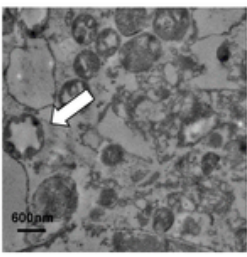

erastin
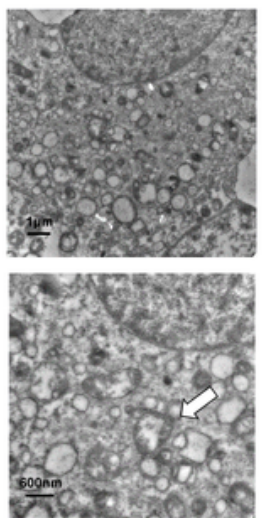

FZKA

Figure 2

FZKA induced ferroptosis in NSCLC cells by FCM and TEM.

A\&B, Cultured A549 and PC9 cells were treated with FZKA $(1.5 \mathrm{mg} / \mathrm{ml})$ for $24 \mathrm{~h}$. Cells were stained with FerroOrange $(1 \mu \mathrm{M})$ for 30 min, the level of $\mathrm{Fe}^{2+}$ lons was detected by flow cytometry, ${ }^{*} p<0.05$. C, Transmission electron microscopy of $\mathrm{H} 1650$ and $\mathrm{H} 1299$ cells treated with DMSO (10 hrs), erastin 
(ferroptosis inducer, $10 \mu \mathrm{M}, 10 \mathrm{hrs}), \mathrm{FZKA}(1 \mathrm{mg} / \mathrm{ml}, 10 \mathrm{hrs})$. Swollen mitochondria with fractured cristae appeared in erastin- and FZKA-treated cells. Scale bar represents $1 \mu \mathrm{m}$ and $600 \mathrm{~nm}$.

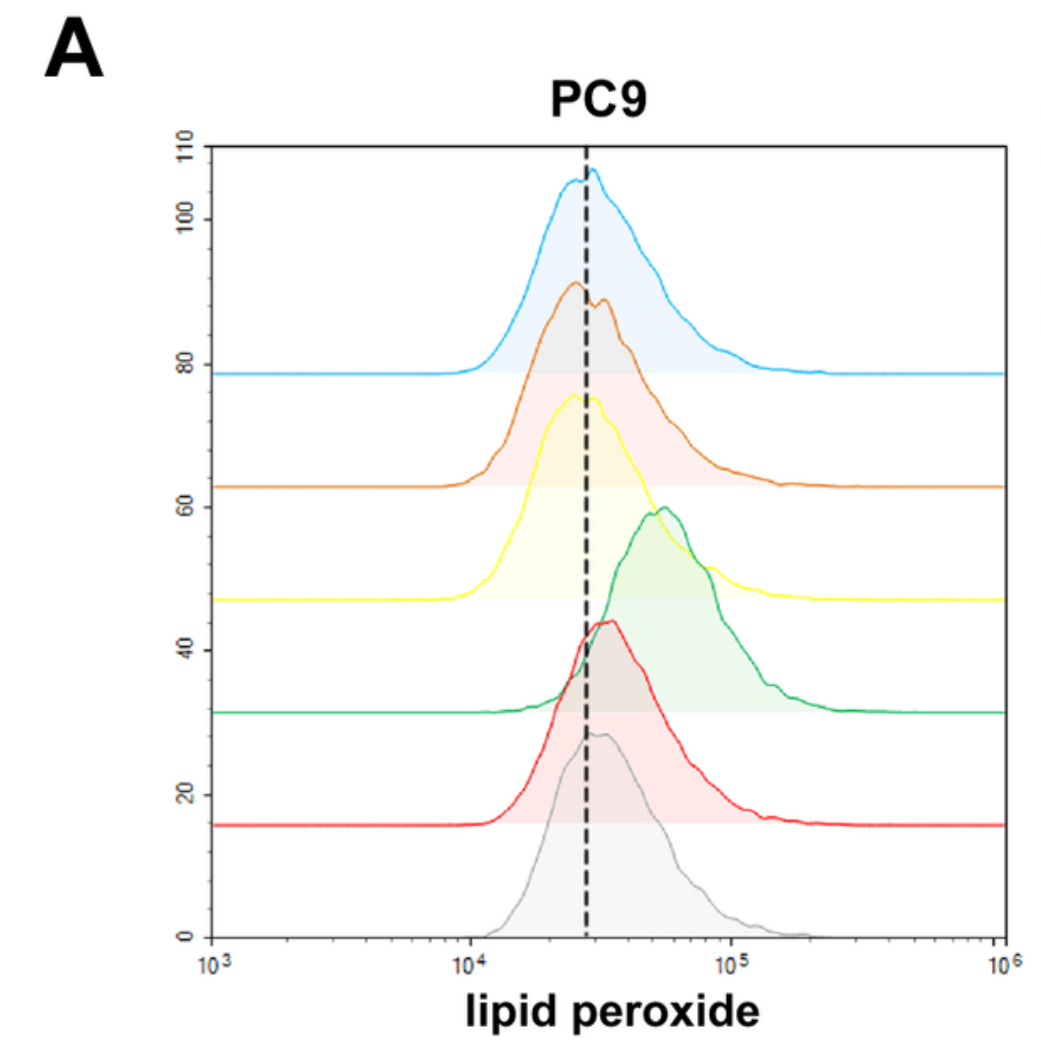

B
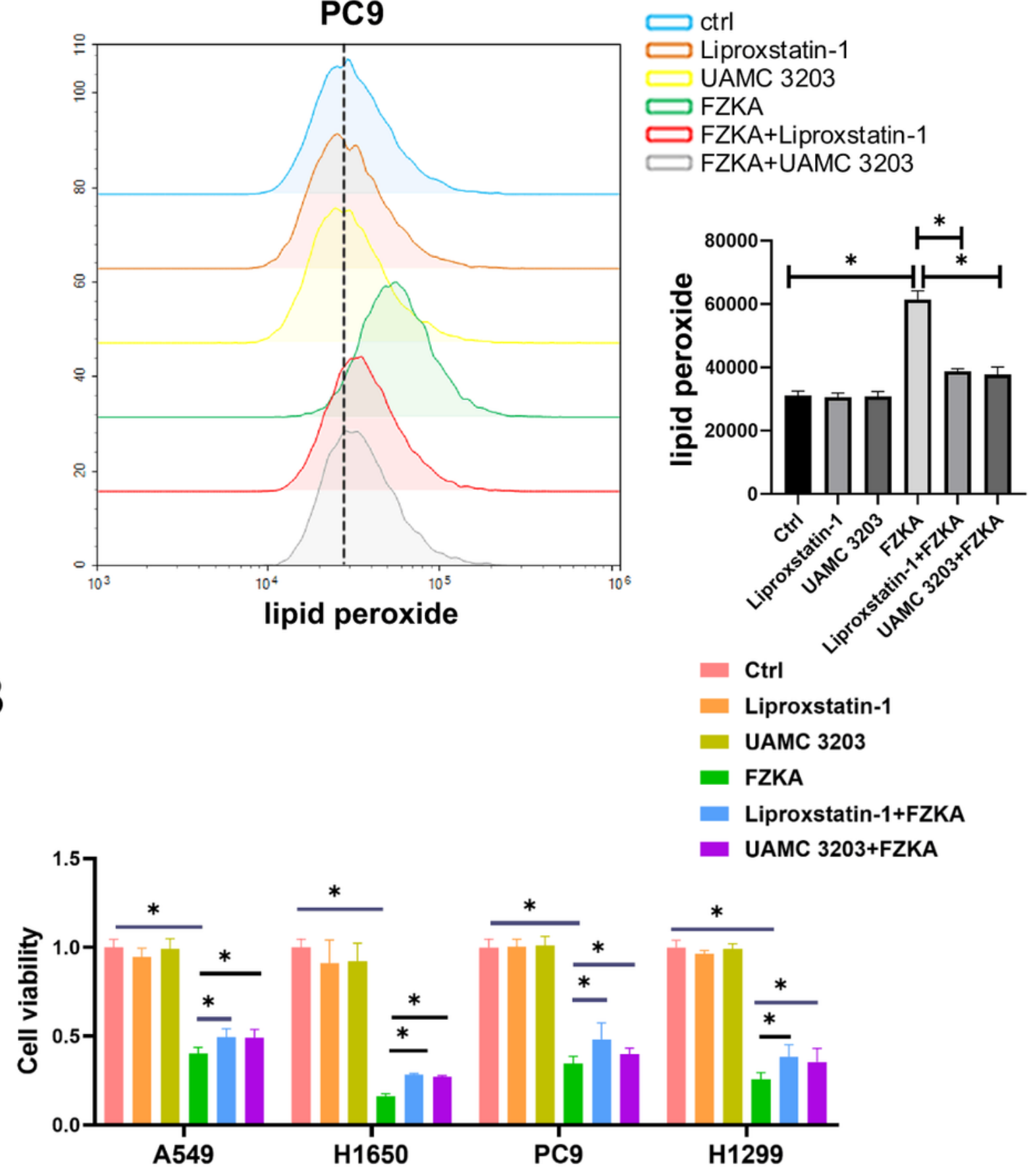

Figure 3

Ferroptosis inhibitors including liproxstatin-1 and UAMC 3203 reversed the effect of FZKA. 
A, PC9 cells were treated as A, and stained with BODIPY ${ }^{\mathrm{TM}} 581 / 591 \mathrm{C} 11(10 \mu \mathrm{M})$ for $30 \mathrm{~min}$. The level of lipid peroxidation was detected by flow cytometry. Each point represents the mean $\pm S E M, n=3,{ }^{\star} p<0.05$. $B$, Cultured NSCLC cells were seeded in 96 well plate, FZKA $(1.5 \mathrm{mg} / \mathrm{mL}$ in A549 and PC9 cells, $1 \mathrm{mg} / \mathrm{mL}$ in $\mathrm{H} 1650$ and H1299 cells), with UAMC3203 (25nM), or liproxstatin-1 (200 nM) for 24h. Cell viability was detected by CCK-8 assay, ${ }^{\star} p<0.05$.

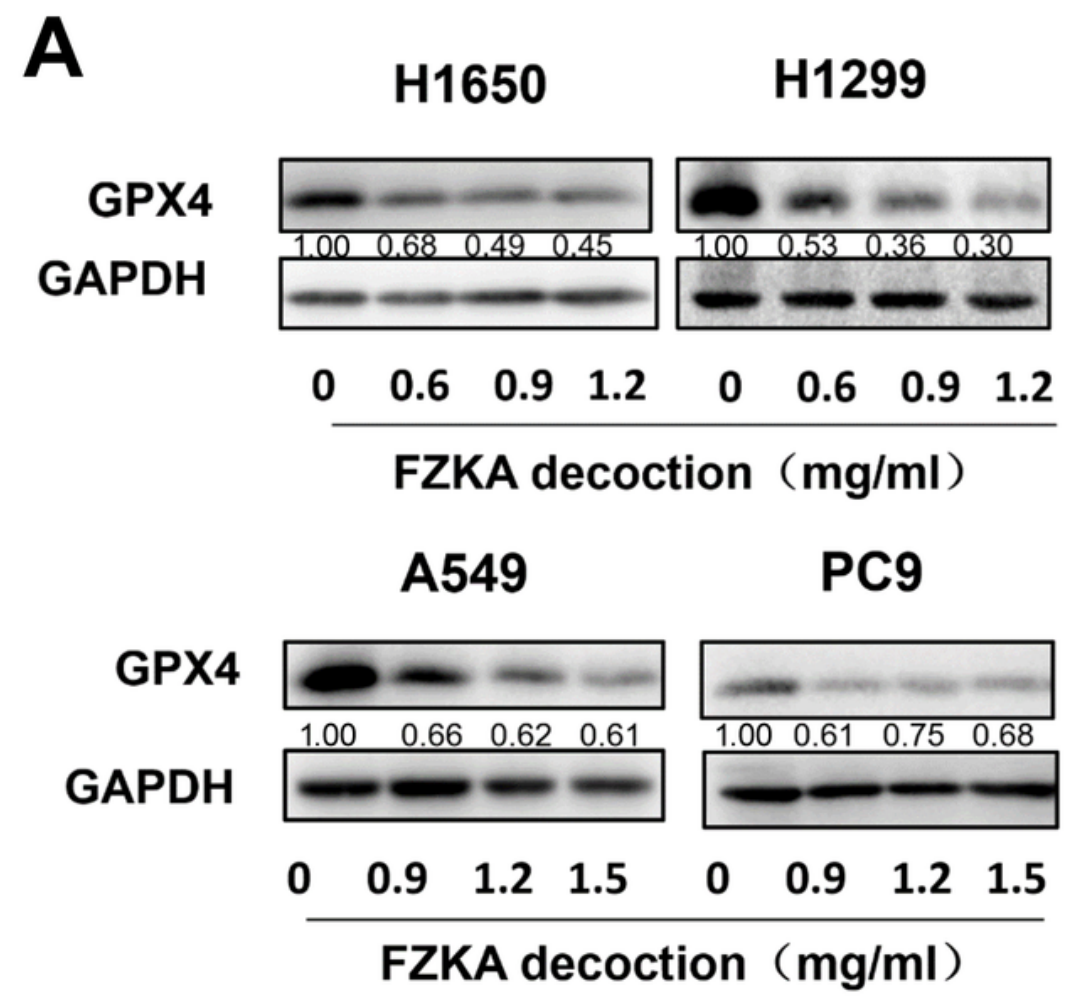

B

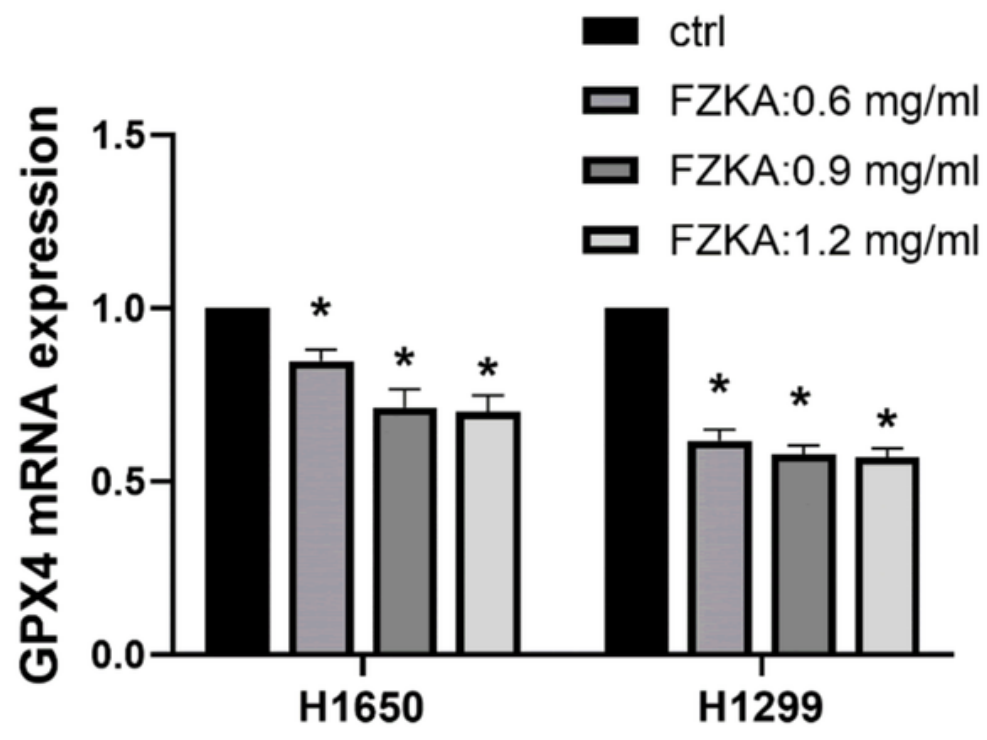

Figure 4 
FZKA downregulated the GPX4 expression in NSCLC cells at both protein and mRNA levels.

A, The protein expression levels of GPX4 were detected by Western blot. B, NSCLC cells were treated with different concentrations of FZKA for 24h. The mRNA expression of GPX4 were detected by qPCR. Each point represents the mean $\pm \mathrm{SEM}, \mathrm{n}=3 .{ }^{*} p<0.05$.

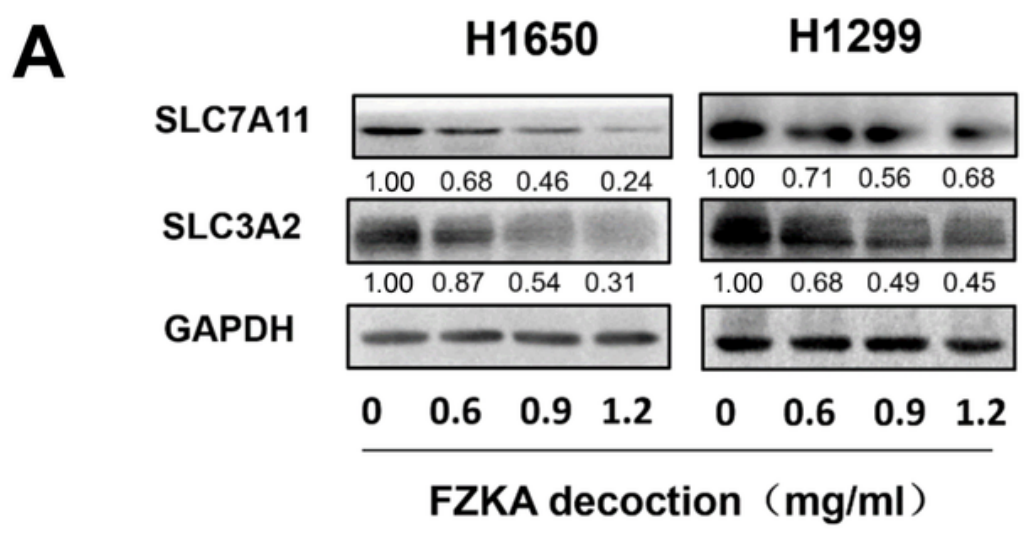

B
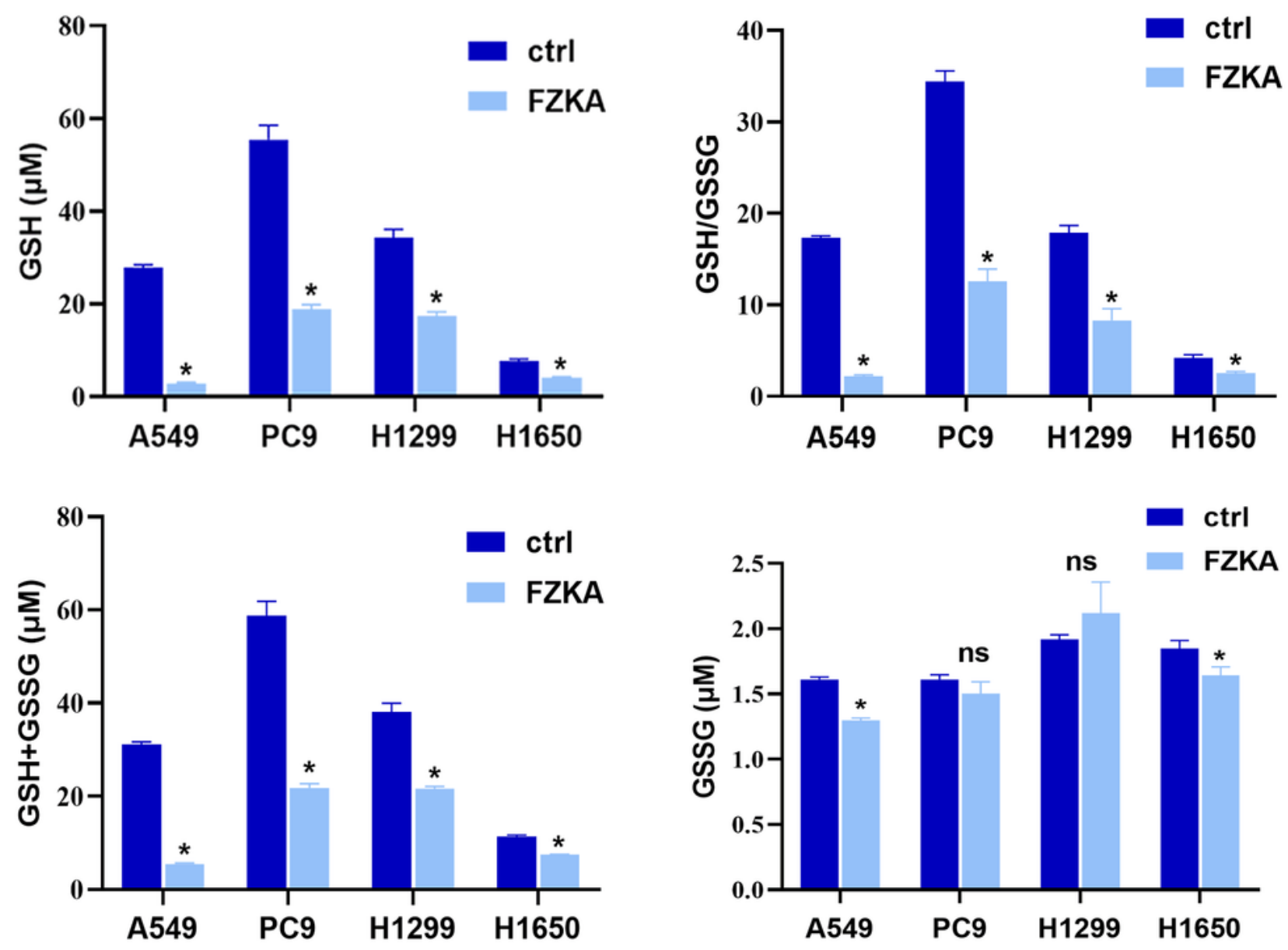

Figure 5 
FZKA decreased the ratio of GSH/GSSG and expression of system xc:.

A, The protein expression levels of SLC7A11 and SLC3A2 were detected by Western blot. B, the levels of GSH and GSSG were measured by GSH and GSSG Assay kit. Each point represents the mean \pm SEM, $n=$ 3. ${ }^{\star} p<0.05$.
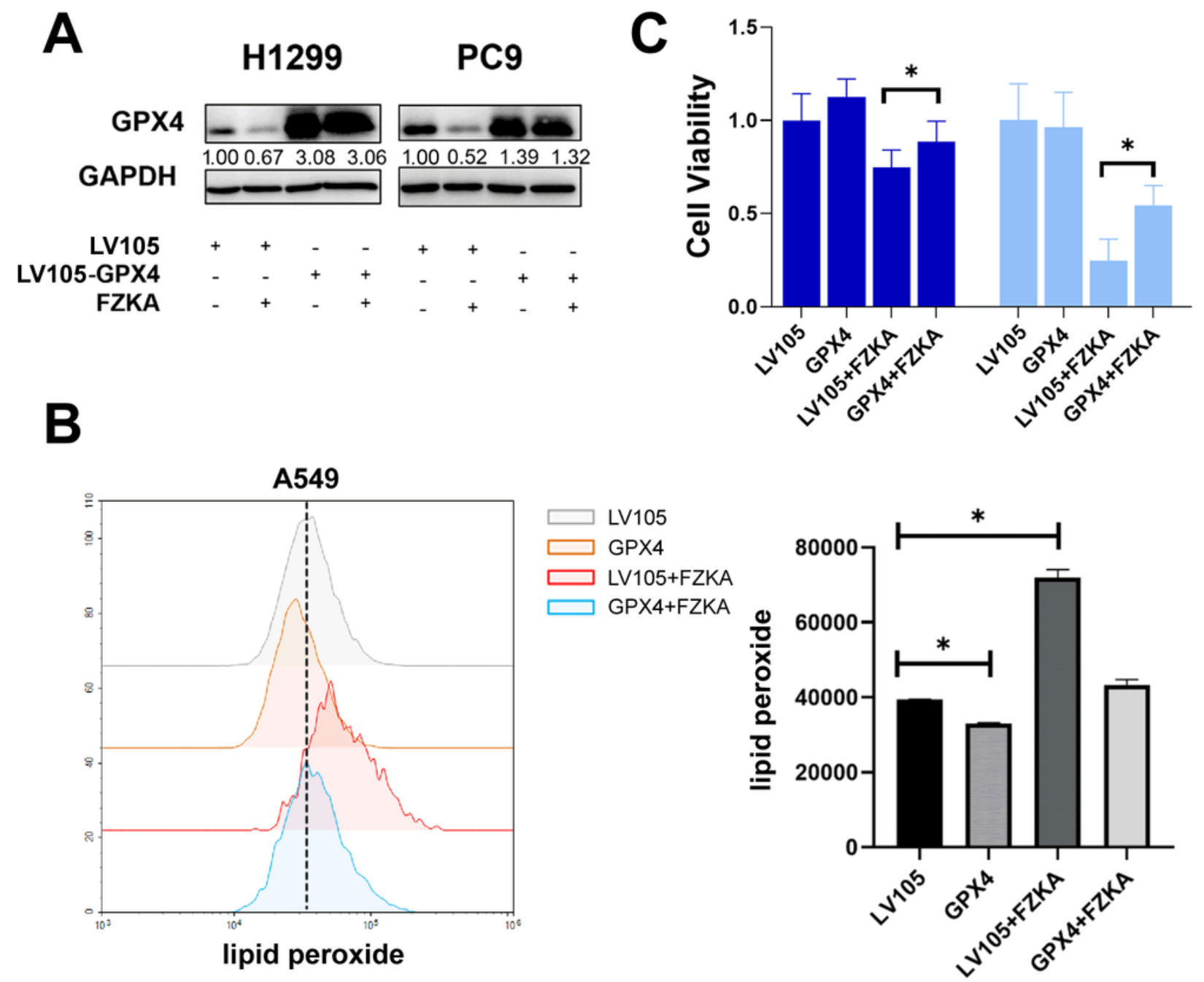

Figure 6

Overexpression of GPX4 reversed the efficacy of FZKA.

A, Cultured H1299 and PC9 cells were transfected with negative control and GPX4 lentiviral vectors, then treated with or without FZKA for $24 \mathrm{~h}$. The expression of GPX4 was detected by western blot. B, lipid peroxidation assay was performed in PC9 cell after treatment with FZKA or/and GPX4 lentivirus. C, Cells were transfected and treated with FZKA, and CCK-8 assay were then conducted. Each point represents the mean $\pm S E M, n=3 .{ }^{*} p<0.05$. 
A

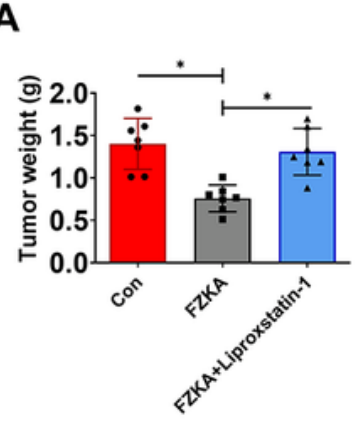

B
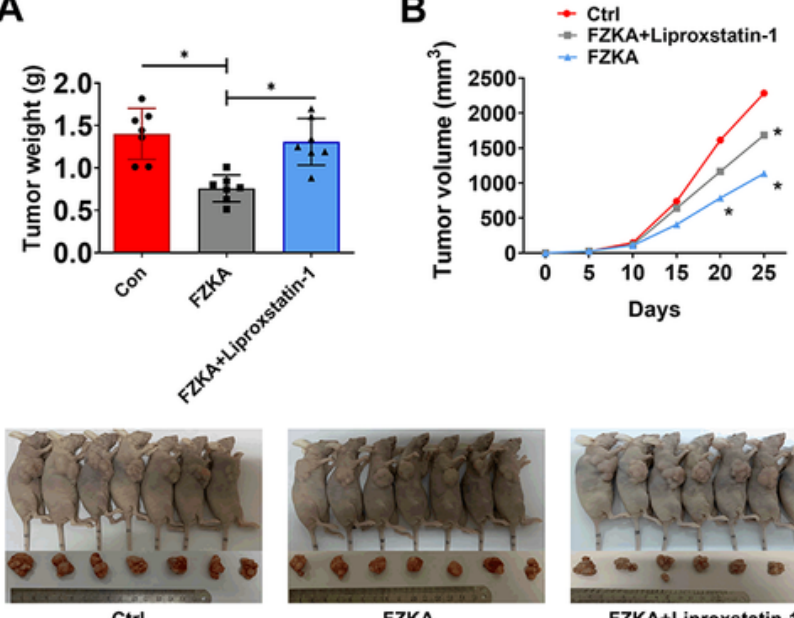

FZKA

C

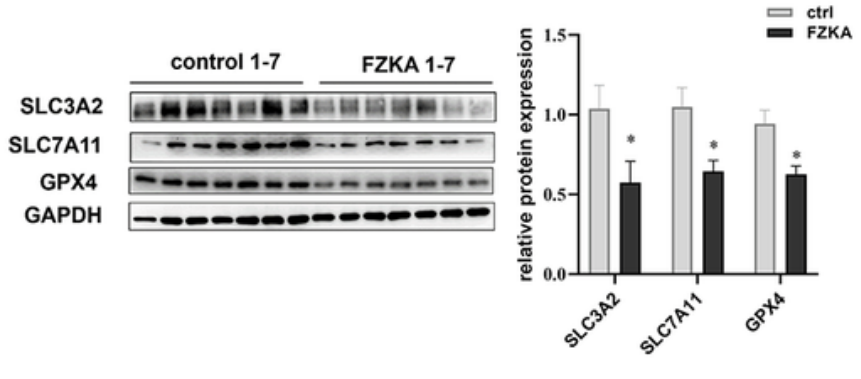

FZKA+Liproxstatin-1
D
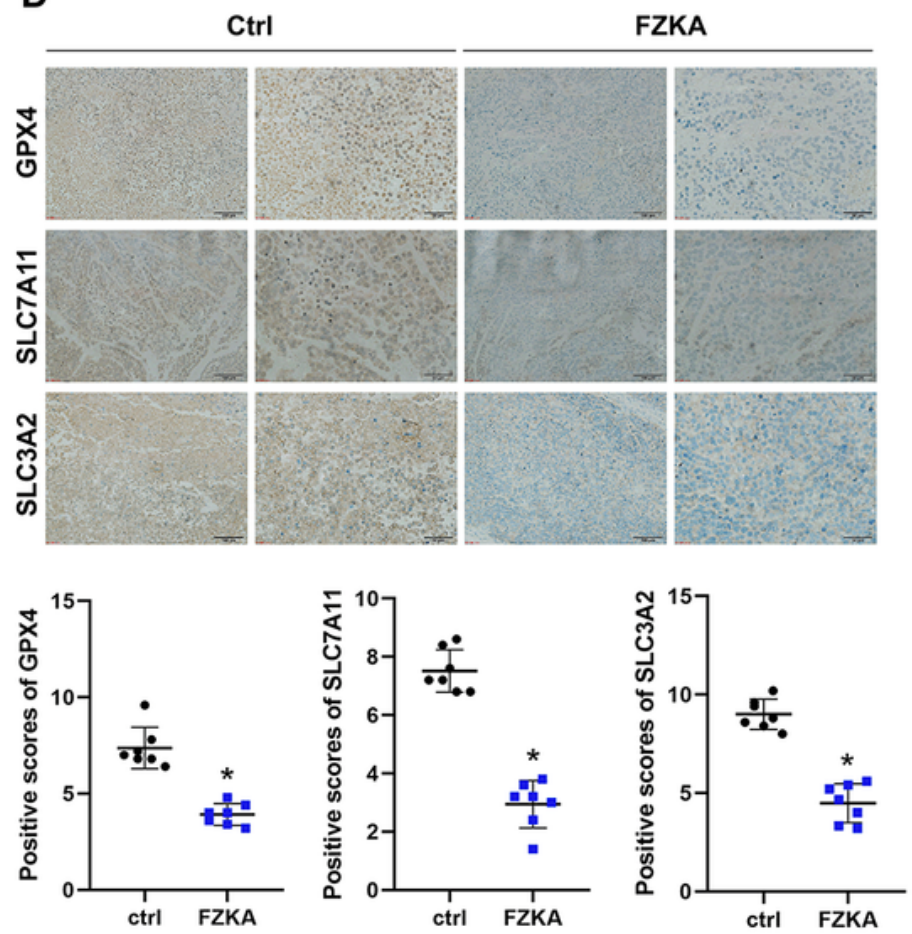

E

FZKA $\longrightarrow$

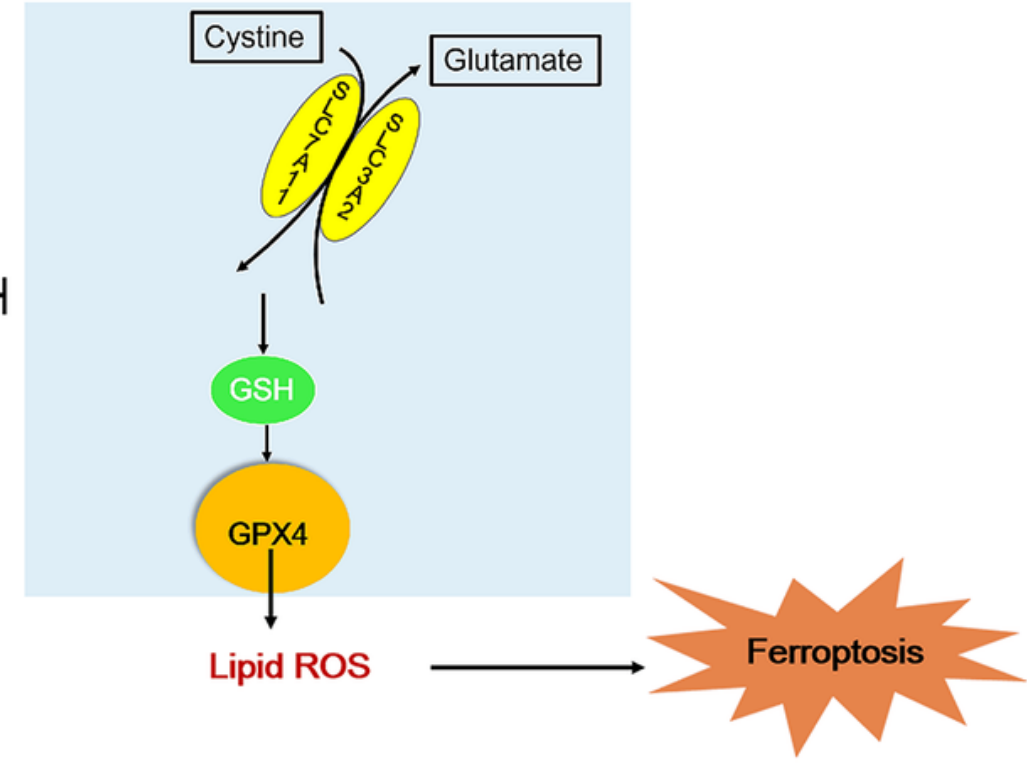

\section{Figure 7}

\section{Validation of FZKA-induced NSCLC cell ferroptosis in vivo.}

A, Mice tumor photograph and tumor weight was showed. Data represents Mean $\pm S E M, n=7$. ${ }^{*}<0.05$. B, Tumor volume in each group was showed. Data represents Mean $\pm \mathrm{SEM}, \mathrm{n}=7$. ${ }^{\star} p<0.05$. C, Western blot analyses of GPX4, SLC7A11 and SLC3A2 expression from tumor tissues. Data represents Mean \pm SEM, $n$ 
$=7 .{ }^{*} p<0.05 . \mathrm{D}$, Immunohistochemistry was carried out to measure the expression of GPX4, SLC7A11 and SLC3A2 in mice tumor tissues. Data represents Mean $\pm \mathrm{SEM}, \mathrm{n}=7$. ${ }^{\star} p<0.05$. $\mathrm{E}$, The diagram showing FZKA induced NSCLC cell ferroptosis through system xc-/GSH/GPX4 axis, and, importantly, GPX4 is the crucial molecular in the process. Finally, inhibition of GPX4 by FZKA leads to NSCLC cell ferroptosis.

\section{Supplementary Files}

This is a list of supplementary files associated with this preprint. Click to download.

- SupplementaryFiguresferroptosis.pdf 